\title{
Radiation source rate estimation through data assimilation of gamma dose rate measurements for operational nuclear emergency response systems
}

\section{Vasiliki Tsiouri* and Spyros Andronopoulos}

Environmental Research Laboratory, Institute of Nuclear Technology and Radiation Protection, National Center for Scientific Research 'DEMOKRITOS', 15310 Agia Paraskevi Attikis, Athens, Greece

E-mail: vtsiouri@ipta.demokritos.gr

E-mail: sandron@ipta.demokritos.gr

*Corresponding author

\section{Ivan Kovalets}

Department of Environmental Modeling, Institute of Mathematical Machine and System Problems, National Academy of Sciences of Ukraine (NASU), pr. Glushkova-42, Kiev 03187, Ukraine E-mail: ik@ucewp.kiev.ua

\section{Leisa L. Dyer}

Safety, Environmental and Radiological Assurance (SERA), Australian Nuclear Science and Technology Organisation (ANSTO), Locked Bag 2001, Kirrawee DC, Sydney 2232, NSW, Australia

E-mail: Leisa.Dyer@ansto.gov.au

\author{
John G. Bartzis \\ Department of Mechanical Engineering, \\ University of Western Macedonia, \\ Bakola \& Sialvera str., 50100, Kozani, Greece \\ E-mail: bartzis@uowm.gr
}

\begin{abstract}
This paper presents an evaluation of an innovative data assimilation method that has been recently developed in NCSR Demokritos for estimating an unknown emission rate of radionuclides in the atmosphere, with real-scale experimental data. The efficient algorithm is based on the assimilation of gamma dose rate measured data in the Lagrangian atmospheric dispersion model DIPCOT and uses variational principles. The DIPCOT model is used in the framework of the nuclear emergency response system (ERS) RODOS. The evaluation is performed by computational simulations of dispersion of Ar-41 that was emitted routinely by the Australian Nuclear Science and Technology Organisation's (ANSTO) previous research reactor, HIFAR, located in Sydney, Australia. In this paper the algorithm is evaluated against a more complicated
\end{abstract}


case than the others used in previous studies: There was only one monitoring station available each day and the site topography is characterised as moderately complex. Overall the estimated release rate approaches the real one to a very satisfactory degree as revealed by the statistical indicators of errors.

Keywords: radiation source rate estimation; data assimilation; variational method; Lagrangian model; nuclear emergency response.

Reference to this paper should be made as follows: Tsiouri, V., Andronopoulos, S., Kovalets, I., Dyer, L.L. and Bartzis, J.G. (2012) 'Radiation source rate estimation through data assimilation of gamma dose rate measurements for operational nuclear emergency response systems', Int. J. Environment and Pollution, Vol. 50, Nos. 1/2/3/4, pp.386-395.

Biographical notes: Vasiliki Tsiouri is a Physicist. In 2011, she defended her $\mathrm{PhD}$ in development of data assimilation methods in atmospheric dispersion models used in emergency response systems in the Department of Mechanical Engineering, University of West Macedonia, Greece. She worked as an Associate Scientist at the Institute of Environmental Research and Sustainable Development (National Observatory of Athens) in atmospheric pollution monitoring and modelling. Since 2005, she is an Associate Scientist at the Institute of Nuclear Technology \& Radiation Protection, NCSR 'Demokritos'. Her main research field is the development and application of nuclear emergency response methods for predicting airborne hazards from accidental releases.

Spyros Andronopoulos is a Researcher at the Environmental Research Laboratory/INTRP of NCSR 'Demokritos'. He received his $\mathrm{PhD}$ in Atmospheric Dispersion from the University of Athens, Greece, in 1994. His research interests are in modelling of atmospheric dispersion, mesoscale prognostic and diagnostic meteorology, meteorological data assimilation, urban air quality and decision-support systems for air pollution situations. He has participated in several European research projects and has published in international journals and conferences.

Ivan Kovalets received his MSc in Applied Physics in 1996 from Moscow Institute of Physics and Technology. In 2001, he defended his $\mathrm{PhD}$ in Computational Fluid Dynamics in the Institute of Hydromechanics of the National Academy of Sciences of Ukraine (NASU). Since 1999, he has been employed as a Research Scientist at the Institute of Mathematical Machine \& System Problems NASU. In 2002 and 2005, he worked in NCSR 'Demokritos' as a Visiting Scientist due to EURATOM fellowships. His today's research interest is data assimilation in meteorological and atmospheric dispersion models, used in emergency response systems.

Leisa L. Dyer received her BSc (hons.) in Applied Mathematics from the University of New South Wales, Sydney, Australia in 2002. She joined ANSTO in 2003 as a computational modeller, and was appointed to an atmospheric scientist in 2007. Her areas of research include transport and land-surface processes in global climate models, isotopic tracers in atmospheric transport, receptor modelling of aerosol data and most recently, evaluating atmospheric dispersion models in complex terrain. She is responsible for the meteorological and environmental gamma monitoring networks at ANSTO and maintaining the emergency response computer system. 
John G. Bartzis is a Professor in the Department of Mechanical Engineering, at the University of West Macedonia, Greece. He obtained his $\mathrm{PhD}$ in Nuclear Engineering from the MIT, USA in 1977. His field of scientific activities includes environmental computational fluid dynamics, indoor and outdoor air pollution, environmental impact of energy production, hydrogen technology and safety. He has participated in or coordinated numerous European research projects in the field of environment. He participates in several international scientific committees. He has authored or co-authored over 150 publications in journals, conference proceedings, books, reports, etc.

This paper is a revised and expanded version of a paper entitled 'Radioactive material release rate estimation through data assimilation of gamma dose rate measurements' presented at the 14th International Conference on Harmonisation within Atmospheric Dispersion Modelling for Regulatory Purposes, Kos, Greece, 2-6 October 2011.

\section{Introduction}

In nuclear power plant accidents that involve release of radionuclides in the atmosphere, the emission rate of radioactive material is usually unknown. During the emergency phase the estimated source term can differ from the true one by the factor of ten or more (US Nuclear Regulatory Commission, 1990). Therefore, improving source rate estimation is of primary importance. A way to assess the release rate is data assimilation (DA) of gamma dose measurements which are typically available around every nuclear power plant. In this respect an innovative computational method has been recently developed in NCSR Demokritos for estimating the unknown emission rate of radionuclides in the atmosphere. The algorithm is based on assimilation of gamma dose rate measured data in the Lagrangian atmospheric dispersion model DIPCOT (Andronopoulos et al., 2009) used in the framework of the nuclear emergency response system (ERS) RODOS (Raskob, 2007) and uses variational principles. The method is described in Tsiouri et al. (2011, 2012). In the latter work (Tsiouri et al., 2012) the method was successfully evaluated against the fluence rate measurements in field experiment of Ar-41 atmospheric dispersion in Mol, Belgium (Drews et al., 2002). In the present work, the method is evaluated against a more complicated case using gamma dose rate measurements from Ar-41 routine releases at the Australian Nuclear Science and Technology Organisation's (ANSTO) previous research reactor, HIFAR, located in Sydney, Australia. The area around the research reactor is characterised by moderately complex topography and spatially varying land cover. The Ar-41 database that is used for the purposes of the study covers various seasons during 2002 to 2003 and includes measured gamma radiation dose rates from four monitoring stations located in a radius of $5 \mathrm{~km}$ around the research reactor. There are 16 days of gamma radiation dose measurements but only one monitoring station is available each day. Therefore, the challenge for improving the source rate in this case is the assimilation of gamma dose rate measured data from only one monitoring station and the complex terrain of dissected plateaus and valleys that surrounds ANSTO. 


\section{Methodology}

\subsection{Model description}

DIPCOT (Andronopoulos et al., 2009) is a three-dimensional model, which simulates atmospheric dispersion estimating particle (puff's) trajectories. It has been comprehensively validated against numerous field and laboratory experiments on atmospheric dispersion (e.g., Andronopoulos and Bartzis, 2010) and it is included in the European real-time, online, decision support (RODOS) system for nuclear emergencies. In DIPCOT there are two modes of particles/puffs movement, the stochastic mode (SM) and the deterministic mode (DM). In DM puffs are transported by the average wind field and grow in size according to well-known Pasquill-type relationships. In SM puffs are transported also by wind fluctuations based on the Langevin equation, formulated for stationary homogeneous isotropic turbulence at the horizontal direction, and on inhomogeneous Gaussian turbulence for the vertical direction, i.e., particles' equations of movement become stochastic. Concentration $C$ (activity concentration of nuclides in air) and gamma dose rates at a particular location and time are calculated by summing the contribution of all neighbouring puffs. A description of the gamma dose calculation methods used in DIPCOT is given in Andronopoulos et al. (2009) and Andronopoulos and Bartzis (2010).

\subsection{DA algorithm}

An innovative and efficient methodology based on variational DA is used for estimating the unknown emission rate (Tsiouri et al., 2011, 2012). The main objective of the DA method is the minimisation of the following cost function with respect to the control vector $\bar{\psi}$ which consists of the source rates corresponding to times of releases of puffs: $\bar{q}$.

$$
\begin{aligned}
& J=J_{1}+J_{2}, J_{1}=\left(\bar{\psi}-\bar{\psi}^{b}\right)^{T} \underline{\underline{B}}^{-1}\left(\bar{\psi}-\bar{\psi}^{b}\right) \\
& J_{2}=\sum_{n=1}^{N_{o}} \sum_{k=1}^{K} \sigma_{O}^{-2}\left(d_{k}^{o}\left(t_{n}\right)-\tilde{d}\left(\bar{r}^{k}\right)\right)^{2}=\left(\bar{d}^{o}-\underline{\underline{G}}\right)^{T} \underline{\underline{O^{-1}}}\left(\bar{d}^{o}-\underline{\underline{G}}\right)
\end{aligned}
$$

Here, $\bar{\psi}^{b}$ is the first guess estimation of the control vector, $\underline{\underline{O}}, \underline{\underline{B}}$ are the covariance matrices of the errors of the observations and the background errors respectively; the vector $\bar{d}^{o} \in R^{N_{o} K}$ consists of the gamma dose rates $d^{o}(n, k)$, measured on each time interval $\Delta t_{n}$ by the $k^{\text {th }}$ station. The elements of $d^{o}$ are ordered sequentially as follows:

$$
d_{l}^{o}=d_{(n-1) K+k}^{o}=d^{o}(n, k)
$$

For substantial improvement in numerical efficiency and accuracy and to enable using the DA method also in the framework of the stochastic Lagrangian atmospheric dispersion models, the control vector reduction technique explained in detail in Tsiouri et al. (2012) is used. This technique is based on the assumption that 
during small enough time interval $\Delta t$, the source rate can be considered as constant with sufficient accuracy. Then the particles could be joined in $P=N_{p} / \Pi$ groups with $\Pi$ particles in each group being characterised by the same source rate: $q_{(j-1) \Pi+1}=q_{(j-1) \Pi+2}=\ldots=q_{j \Pi}=\tilde{q}_{j}, 1 \leq j \leq P$. Here $\tilde{q}_{j}$ are the values characterising the source rate of the $j^{\text {th }}$ group of particles, which form the reduced control vector: $\underline{\tilde{q}}$ of size

$P$. Clearly, the value of $P$ depends on the choice of the time interval $\Delta t$ during which the source rate could be considered as constant and thus it is a free variable that depends on the expert judgement of the user. Note that if $P=1$, then the source rate is assumed to be constant during the whole release interval. Instead of initial problem of minimising equation (1) with respect to the control vector $\bar{q}$ consisting of the release rates of individual particles the 'reduced' minimisation problem is solved in which the same function is minimised with respect to the reduced control vector $\underline{\tilde{q}}$. The cost function [equation (1)] with constraint of positive control vector values is minimised using the IMSL ${ }^{\circledR}$ package (IMSL MATH/LIBRARY, 1987).

\section{Applications - results}

In the present work, DA runs are performed and the DA algorithm is evaluated against the measured release rate from the Ar- 41 database developed at the ANTSO previous research reactor, HIFAR, located in Sydney, Australia. Specifically, 16 different cases are simulated that cover winter and summer periods of the years 2002 and 2003 and include all the atmospheric stability conditions. The Ar-41 database used for the method evaluation include the Ar-41 stack emission rate, measured meteorological data from two stations and measured gamma dose rates from four monitoring stations located in a radius of $5 \mathrm{~km}$ around the reactor. All the above data were available in 15-min time intervals. The terrain elevation and the land cover were available on a grid of $25 \mathrm{~m}$ resolution for the area of interest around the site. Figure 1 shows the computational domain with terrain elevation contours, the Ar-41 release location, the meteorological stations and the gamma dose rate detectors. The terrain is moderately complicated with hills of about $190 \mathrm{~m}$ height and a valley that transverses the domain. The land cover is varying, including urban (south-east part), suburban (central part), woods (along the river) and low vegetation (north and south-west part) areas. The available meteorological measurements included wind speed, wind direction and temperature at the levels of 10 and $49 \mathrm{~m}$ for the station Met00 and wind speed and direction at $18.5 \mathrm{~m}$ for the station Met01 (Figure 1). The atmospheric stability has been determined by the pre-processors for each 15 -min time interval from the temperature gradient between 10 and $49 \mathrm{~m}$ and from the wind speed. The raw wind velocity data of $1 \mathrm{~min}$ sampling at heights $69 \mathrm{~m}$ and $78 \mathrm{~m}$ have been averaged on 10-min intervals to drive the dispersion model, together with the Pasquill-Gifford stability categories given in 10-min intervals in the database. The meteorological data were pre-processed by the meteorological pre-processor FILMAKER of the RODOS system to prepare input meteorological fields for the DIPCOT model.

Simulations with the DIPCOT model have been performed with the following set of parameters. The puffs were released at a time interval $\tau \approx 2 s$ and at a time interval $\tau \approx 4 s$. Simulations have been performed in the SM of DIPCOT operation. The first guess source 
emission rate was set by a factor of 10 greater than the true rate. Different number of source time intervals (parameter $P$ of CVR technique) has been used in different runs. A detailed description of the simulations is given in Table 1.

Figure 1 The computational domain with terrain elevation contours, the Ar-41 release location, the meteorological stations (Met00, Met01) and the gamma dose rate detectors (Det 9, Det 16, Det 17, Det 18) (see online version for colours)

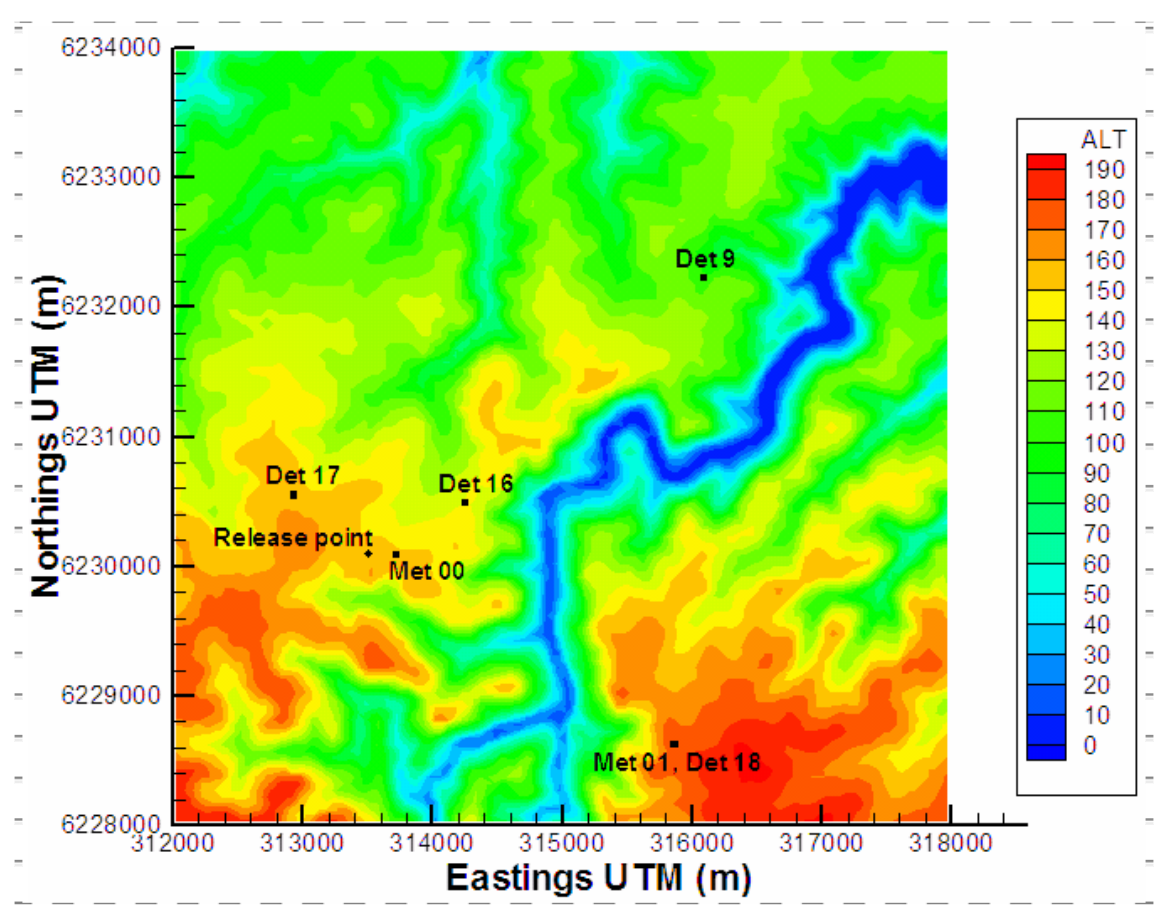

Table 1 The detailed description of the simulations

\begin{tabular}{|c|c|c|c|c|c|}
\hline Case (date) & Station name & $\begin{array}{l}\text { Detector } \\
\text { point } \\
\text { (station \#) }\end{array}$ & $\begin{array}{c}\text { Distance } \\
\text { from } \\
\text { HIFAR } \\
(\mathrm{km})\end{array}$ & Case details & $\begin{array}{l}\text { Tested value of } \\
\text { P/corresponding values of } \\
\text { time intervals }(\Delta t) \text { during } \\
\text { which the source rate is } \\
\text { assumed to be constant }\end{array}$ \\
\hline $\begin{array}{l}\text { Day } 1 \\
(06 / 06 / 2003)\end{array}$ & $\begin{array}{c}\text { Waste } \\
\text { services } \\
\text { (WS) }\end{array}$ & 17 & 0.73 & $\begin{array}{l}\text { Winter, } \\
\text { unstable } \\
\text { conditions }\end{array}$ & $\begin{array}{c}1,2 \text { and } 3 / \Delta t \sim \\
450,225,150 \mathrm{~min}\end{array}$ \\
\hline $\begin{array}{l}\text { Day } 2 \\
(17 / 12 / 2002)\end{array}$ & $\begin{array}{l}\text { Main gate } \\
(\mathrm{MG})\end{array}$ & 16 & 0.82 & $\begin{array}{l}\text { Summer, stable } \\
\text { conditions }\end{array}$ & $\begin{array}{l}1,2 \text { and } 3 / \Delta t \sim \\
180,60,30 \mathrm{~min}\end{array}$ \\
\hline $\begin{array}{l}\text { Day } 3 \\
(22 / 06 / 2003)\end{array}$ & $\begin{array}{l}\text { Boys town } \\
\text { (BT) }\end{array}$ & 18 & 2.78 & $\begin{array}{l}\text { Winter, stable } \\
\text { conditions }\end{array}$ & $\begin{array}{c}1,2 \text { and } 3 / \Delta t \sim \\
405,202,5,135 \text { min }\end{array}$ \\
\hline $\begin{array}{l}\text { Day } 4 \\
(09 / 07 / 2003)\end{array}$ & $\begin{array}{l}\text { Barden ridge } \\
\text { (BR) }\end{array}$ & 9 & 3.33 & $\begin{array}{l}\text { Winter, stable } \\
\text { conditions }\end{array}$ & $\begin{array}{c}1,2 \text { and } 3 / \Delta t \sim \\
210,105,70 \mathrm{~min}\end{array}$ \\
\hline
\end{tabular}

Figure 2 shows how efficient are the DIPCOT model in estimating the unknown source rate with the implementation of the DA algorithm. This figure presents the source emission rate estimations as result of the assimilation of gamma dose rate data for the 
cases of day 1, day 2, day 3 and day 4 in case of the stochastic version of DIPCOT against time. The time in $\mathrm{x}$-axis is the time of release. The puffs were released at a time interval $\tau \approx 2 s$. The source rate is estimated at fixed intervals over the release period of time depending on the tested value of $P$. Results with different number of groups $P$ are presented. The true source rate is known but we supposed we do not know it and an arbitrary value of it was taken (ten times more). The 'first guess' source rate and the true source rate are not constant (first guess is ten times more than true). The other lines represent the estimated source rate for different values of $P$. As already explained in the section of methodology the value of $\mathrm{P}$ depends on the choice of the time interval $\Delta t$ during which source rate is considered as constant (if $P=1$, then the source rate is assumed to be constant during the whole release interval).

Figure 2 Release rate estimations as result of gamma dose rate assimilation, for

(a) day 1 - WS station (Det 17), (b) day 2 - MG station (Det 16),

(c) day 3 - BT station (Det 18) and (d) day 4 - BR station (Det 19) cases

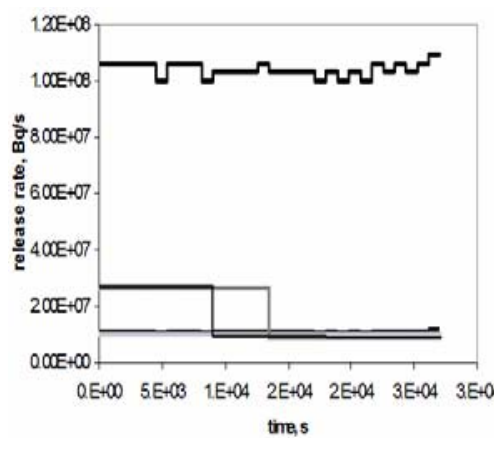

(a)

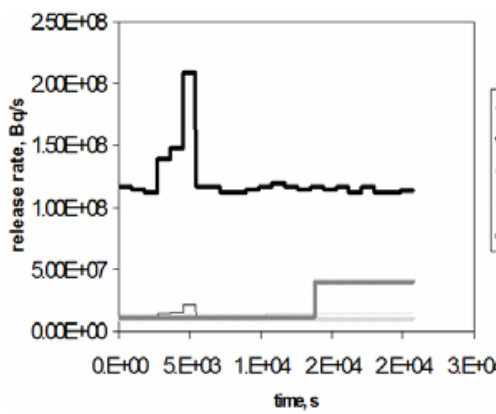

(c)

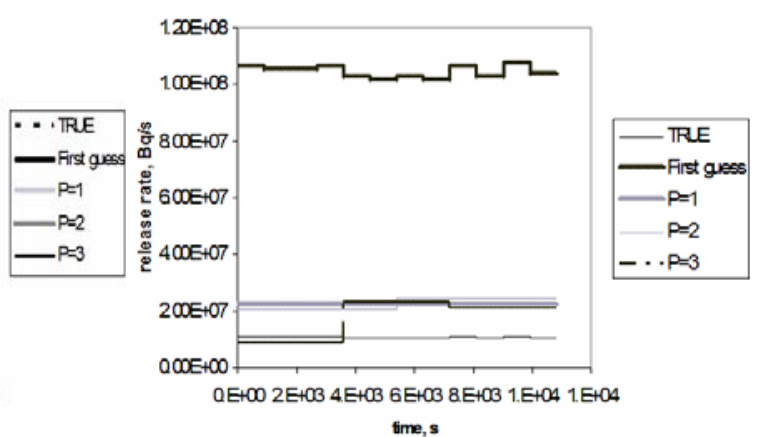

(b)

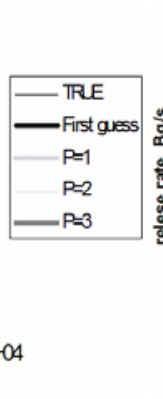

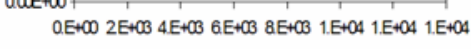

fime, $s$

(d)

As it can be clearly seen from Figure 2 the algorithm managed to estimate the unknown source rate. The adjusted source functions in all cases are much better than the first guess source function. Overall the estimated release rate approaches the real one to a very satisfactory degree for day 1 (Det 17), day 2 (Det 16) and day 3 (Det 18) cases under all stability conditions. For day 4 (Det 9) because of the small sample size the results are less satisfactory, but even in this worst case the algorithms improves the source rate.

It is important to mention that the complexity of each case tested is increased by the complexity of terrain. The complexity of terrain results in more difficult accurate calculations in forward run of the model and consequently it increases difficulty of 
assimilation problem. Additionally, the difficulty of assimilation problem is increased as the number of available monitoring stations is decreased. In the present work, one monitoring station was available each day and the site topography is characterised as moderately complex. Therefore, even with assimilation of gamma dose rate measured data from only one monitoring station and moderately complex site topography the DA method allows for substantial improvement of source rate.

Table 2 Mean absolute relative error (MAE) and mean relative biases (MRB) of calculated source function as compared to measured source function

\begin{tabular}{ccccccc}
\hline Case & Station name & $\begin{array}{c}\text { Detector } \\
\text { point } \\
\text { station \# }\end{array}$ & $\begin{array}{c}\text { No. of } \\
\text { puffs }\end{array}$ & $P$ & MAE & MRB \\
\hline Day 1 & Waste services (WS) & 17 & 13,500 & First guess & 9.0 & 9.0 \\
Day 1 & Waste services (WS) & 17 & 13,500 & 3 & 0.62 & 0.46 \\
Day 1 & Waste services (WS) & 17 & 13,500 & 2 & 0.86 & 0.71 \\
Day 1 & Waste services (WS) & 17 & 13,500 & 1 & 0.06 & -0.06 \\
Day 1 & Waste services (WS) & 17 & 6,300 & First guess & 9.0 & 9.0 \\
Day 1 & Waste services (WS) & 17 & 6,300 & 3 & 1.3 & 1.14 \\
Day 1 & Waste services (WS) & 17 & 6,300 & 2 & 1.12 & 1.02 \\
Day 1 & Waste services (WS) & 17 & 6,300 & 1 & 0.02 & -0.01 \\
Day 2 & Main gate (MG) & 16 & 5,400 & First guess & 9.0 & 9.0 \\
Day 2 & Main gate (MG) & 16 & 5,400 & 3 & 0.80 & -0.67 \\
Day 2 & Main gate (MG) & 16 & 5,400 & 2 & 1.12 & 1.12 \\
Day 2 & Main gate (MG) & 16 & 5,400 & 1 & 1.12 & 1.12 \\
Day 3 & Boys town (BT) & 18 & 10,344 & First guess & 9.0 & 9.0 \\
Day 3 & Boys town (BT) & 18 & 10,344 & 3 & 0.88 & 0.70 \\
Day 3 & Boys town (BT) & 18 & 10,344 & 2 & 0.28 & -0.09 \\
Day 3 & Boys town (BT) & 18 & 10,344 & 1 & 0.17 & -0.17 \\
Day 4 & Barden ridge (BR) & 9 & 6,300 & First guess & 9.0 & 9.0 \\
Day 4 & Barden ridge (BR) & 9 & 6,300 & 2 & 4.58 & 4.58 \\
Day 4 & Barden ridge (BR) & 9 & 6,300 & 1 & 4.11 & 4.11 \\
\hline Note: Er & of the first gus soun & & 2 & \\
\hline
\end{tabular}

Note: Errors of the first guess source function as well as the errors of source functions corrected in assimilation runs with different values of CVR parameter $P$.

This qualitative result is confirmed with the results of the mean relative absolute error (MAE) and the mean relative bias (MRB) presented in the Table 2 $\left(M A E=\left\langle\left|q^{a}-q^{t}\right|\right\rangle /\left\langle q^{t}\right\rangle, \mathrm{MRB}=\left\langle q^{a}-q^{t}\right\rangle /\left\langle q^{t}\right\rangle\right)$, where $q$ is the source function, \langle\rangle means averaging, superscripts ' $a$ ' and ' $t$ ' denote the analysed and the true source function respectively). The results obtained by the stochastic version of DIPCOT at a time interval $\tau \approx 2 s$ for day 1 , day 2 , day 3 and day 4 cases and with different values of the CVR parameter $\mathrm{P}$ are presented in the Table 2 . The results by setting the time interval that the puffs were released to $\tau \approx 4 s$ are also presented for the day 1 . Generally, as follows from these results in all cases the analysed source rate in the assimilation runs is much better than the first guess function even if in the forward run (e.g., day 4) the model did not succeed in attaining the suggested satisfactory performance as reported in Andronopoulos 
et al. (2010). Satisfactory results also obtained even if we reduce the no. of puffs to half as it can be easily seen in Table 2 (for day 1 case).

\section{Conclusions}

The innovative and efficient DA method that has been recently developed in NCSR Demokritos for estimating an unknown emission rate of radionuclides in the atmosphere is evaluated using gamma dose rate measurements from $\mathrm{Ar}-41$ routine releases at the ANTSO previous research reactor, HIFAR, located in Sydney, Australia. The area around the research reactor is characterised by moderately complex topography and spatially varying land cover. The Ar-41 database that is used for the purposes of the study covers various seasons during 2002 to 2003 . The method is based on the assimilation of gamma dose rate measured data in the Lagrangian stochastic atmospheric dispersion model DIPCOT and uses variational principles. The DIPCOT model is used in the framework of the nuclear ERS RODOS. In the DA runs performed in this study, the first guess source emission rate has been set by a factor of 10 greater than the true one. In all cases of DA runs the statistical indicators of errors of the estimated source emission rate as compared to the measured one were reduced. In all cases the estimated release rate approaches the real one to a very satisfactory degree as revealed by the statistical indicators of errors under all stability conditions. Even for the day 4 (Det 9) with small sample size the algorithm improves the source rate. The DA method is successfully evaluated against a complicated case, under a range of atmospheric stability conditions. There was only one monitoring station available each day, therefore even with assimilation of gamma dose rate measured data from only one monitoring station the DA method allows for substantial improvement of source rate. Therefore, the presented results demonstrate the potential of the developed DA algorithm for application in operational nuclear ERSs.

\section{References}

Andronopoulos, S. and Bartzis, J.G. (2010) 'Gamma radiation dose calculation method for Lagrangian-puff atmospheric dispersion models used in real-time emergency response systems', Journal of Radiological Protection, Vol. 30, No. 4, pp.747-759.

Andronopoulos, S., Davakis, S. and Bartzis, J. (2009) 'RODOS DIPCOT model description and evaluation', RODOS Report RODOS(RA)-TN(09)-01, 27p, available at http://www.rodos.fzk. de/Documents/Public/HandbookV6f/Volume3/RA2TN0901_DIPCOT.pdf (accessed on 21 August 2011).

Andronopoulos, S., Dyer, L.L., Davakis, E. and Bartzis, J.G. (2010) 'Evaluation of dispersion models DIPCOT and RIMPUFF used in decision support systems for nuclear and radiological emergency response', Proceedings of the 13th International Conference on Harmonisation within Atmospheric Dispersion Modelling for Regulatory Purposes, pp.143-147.

Drews, M. et al. (2002) 'Measurements of plume geometry and Argon-41 radiation field at the BR1 reactor in Mol, Belgium', Report of NKS project NKS/BOK-1, ISBN 87-7893-109-6, NKS Secretariat, available at http://www.nks.org/en/nks reports/view_document.htm?id= 111010111119804 (accessed on 27 March 2011).

IMSL MATH/LIBRARY (1987) User's Manual, Vols. 1-3, (QA76.73 in NIST Gaithersburg Research Information Center, Admin E-120) IMSL Inc., Houston, TX.

Raskob, W. (2007) 'European approach to nuclear and radiological emergency management and rehabilitation strategies (EURANOS)', Kerntechnik, Vol. 72, No. 4, pp.172-175. 
Tsiouri, V., Kovalets, I., Andronopoulos, S. and Bartzis, J. (2011) 'Development and first tests of a data assimilation algorithm in a Lagrangian puff atmospheric dispersion model', International Journal of Environment and Pollution, No. 44, pp.147-155.

Tsiouri, V., Kovalets, I., Andronopoulos, S. and Bartzis, J. (2012) 'Emission rate estimation through data assimilation of gamma dose measurements in a Lagrangian atmospheric dispersion model', Radiation Protection Dosimetry, Vol. 148, No. 1, pp.34-44.

US Nuclear Regulatory Commission (1990) Analysis of CDF from Internal Events: Expert Judgment, Report NUREG/CR-4550, April, Vol. 2, Washington, DC. 